\title{
Blocking despite changes in reinforcer identity
}

\author{
BEN A. WILLIAMS \\ University of California, San Diego, La Jolla, California
}

\begin{abstract}
Rats were trained on a discriminated operant barpressing task according to a standard blocking design. In some conditions, the reinforcer was changed between the pretraining and compound conditioning phases; for other conditions, the reinforcer remained the same across phases. In three separate experiments using both between- and within-subject designs, strong blocking effects occurred regardless of the change in the reinforcer. In a fourth experiment, a multiple schedule of reinforcement was used in which response-independent reinforcers were superimposed on the schedule of response-contingent reinforcers. The degree of response suppression caused by the free reinforcer was greater when the free reinforcers were the same as the response-contingent reinforcers than when they were different. The role played by the reinforcer identity in contingency experiments thus appears to be different from the role it plays in blocking experiments.
\end{abstract}

Conditioning to one element (B) of a compound stimulus $(\mathrm{AB})$ is often blocked if the other element of that compound (A) has previously been paired with the reinforcer (Kamin, 1968). Blocking may fail to occur, however, when the reinforcer is changed between the initial pretraining with the A element alone and the compound stimulus training (e.g., Stickney \& Donahoe, 1983), even when the change involves a decrease in the potency of the reinforcer (e.g., Dickinson, Hall, \& Mackintosh, 1976). This finding of unblocking with downshifts in the value of the reinforcer has sponsored the view that conditioning occurs when the subject is "surprised" by the particular reinforcer that is presented.

Studies of unblocking have provided one of several sets of evidence that conditioning is affected not only by the value of the reinforcer, but also by its specific identity. Other evidence for the role of such specificity comes from procedures in which the reinforcer is altered in some way after conditioning has occurred, with the result that the conditioned response produced by that reinforcer is differentially affected despite never having occurred in conjunction with that altered reinforcer (Colwill \& Rescorla, 1986; Delamater \& LoLordo, 1991).

Given the general theoretical significance of these findings, it is important to recognize that unblocking has not occurred in all studies (e.g., Bakal, Johnson, \& Rescorla, 1974; Mackintosh \& Cotton, 1985; Wagner, Mazur, Donegan, \& Pfautz, 1980). It thus becomes of interest to determine the specific characteristics of the reinforcer that must be changed in order for the unblocking effect to occur. In other words, what are the dimensions of reinforcer identity that are critical in determining when the animal will or will not be "surprised." Alternatively, what deter-

This research was supported by NSF Grant BNS-9010323. Requests for reprints should be addressed to the author, Department of Psychology, University of California, San Diego, La Jolla, CA 92093-0109 (e-mail: bawilliams@ucsd.edu). mines when blocking and unblocking will occur if "surprise" is not the appropriate explanatory framework?

Ganesan and Pearce (1988) have recently reported failures to disrupt blocking that appear to cause substantial difficulty for any interpretation based on the surprise concept. Using a standard blocking design with magazine entry as the conditioned response, water or food served as the reinforcer, which was either changed or unchanged between the pretraining and compound conditioning phases. Typical blocking effects were obtained when the same reinforcer was used in both phases. Most importantly, blocking was unaffected when the reinforcer in the two phases was changed from water to food, or vice versa.

To explain their results, Ganesan and Pearce argued, following Konorski (1967), that it was important to distinguish between two different representations of the unconditioned stimulus (US), one being a general representation that contains information about the affective properties of the reinforcer, the other being more specific, representing the reinforcer's sensory attributes. Since water and food have very different sensory qualities, the implication is that blocking must be affected primarily by the general affective properties of the reinforcer. Thus, whenever those affective properties remain the same, blocking will occur, despite changes in other specific characteristics of the particular reinforcing event.

Although Konorski's distinction between the different properties of the reinforcer (also see Wagner \& Brandon, 1989) provides an explanation of Ganesan and Pearce's (1988) failure to disrupt blocking, it is difficult to reconcile those results with the high degree of reinforcer specificity obtained with reinforcer devaluation procedures (e.g., Colwill \& Rescorla, 1986). For example, when response A is paired with outcome $\mathrm{X}$ and response $\mathrm{B}$ is paired with outcome $\mathrm{Y}$, devaluing $\mathrm{X}$ produces a strongly differential reduction in the level of response A. Given that the two reinforcers are usually chosen to be similar in hedonic value, they presumably share 
the same affective representation according to Konorski's typology. The fact that differential effects on the two responses nevertheless occur thus implies that it is the specific sensory properties of the reinforcer, rather than its general affective properties, that are responsible for the maintenance of the instrumental response. The issue raised is why the corresponding sensory properties of the reinforcer were not also responsible for the behavior in the procedure of Ganesan and Pearce (1988).

One possible resolution of this puzzle is that the role of reinforcer specificity is different in Pavlovian and instrumental learning procedures. That is, the general affective quality of the reinforcer may be critical for Pavlovian conditioning, while the specific sensory qualities may be more important for instrumental conditioning. It thus becomes important to determine whether failures to produce unblocking may also occur with instrumental conditioning when significant changes in the reinforcer are made between the pretraining and compound stimulus phases. Experiments 1-3 of the present report investigated that issue.

\section{EXPERIMENT 1}

Experiment 1 was a conceptual replication of Ganesan and Pearce (1988), except that a typical discretetrial operant barpressing task was used. A standard blocking design was used in which leverpressing was reinforced during discrete 5 -sec stimulus periods (stimulus A), separated by intertrial intervals (ITI) in which reinforcement was unavailable. The outline of the procedure is shown in Table 1. After strong stimulus control over leverpressing had been established for stimulus A, a second stimulus (B) was compounded with $\mathrm{A}(\mathrm{A}+\mathrm{B}$ compound) and training on the discrete-trial procedure was continued. For some subjects, the reinforcer used during pretraining was also used during the compound conditioning phase (Groups blocking-same). For other subjects, the reinforcer was switched to the alternative reinforcer during compound conditioning (Groups blocking-different). Control conditions received no discrimination training during Phase 1 (Groups control). All groups were tested for control by stimulus B after compound conditioning had been completed. At issue

Table 1

Outline of the Procedure of Experiment 1

\begin{tabular}{|c|c|c|c|}
\hline Group & Phase 1 & Phase 2 & Test \\
\hline \multicolumn{4}{|l|}{ Blocking } \\
\hline \multicolumn{4}{|l|}{ Same } \\
\hline Sucrose & A-sucrose & $(\mathrm{A}+\mathrm{B})$-sucrose & $\mathrm{B}, \mathrm{A}$ \\
\hline Chow & A-chow & $(\mathrm{A}+\mathrm{B})$-chow & " \\
\hline \multicolumn{4}{|l|}{ Different } \\
\hline Sucrose & A-chow & $(\mathrm{A}+\mathrm{B})$-sucrose & $"$ \\
\hline Chow & A-sucrose & $(\mathrm{A}+\mathrm{B})$-chow & $"$ \\
\hline \multicolumn{4}{|l|}{ Control } \\
\hline Sucrose & No training & $(\mathrm{A}+\mathrm{B})$-sucrose & $\mathrm{B}, \mathrm{A}$ \\
\hline Chow & No training & $(\mathrm{A}+\mathrm{B})$-chow & " \\
\hline
\end{tabular}

Note-Stimuli A and B were either a tone or a clicker, counterbalanced across subjects in each group. was whether blocking caused by the initial pretraining would be affected by changing the reinforcer between phases.

\section{Subjects}

A total of 54 male Sprague-Dawley albino rats (obtained from Bantin Kingman), ranging in age from 3 to 8 months at the start of the study, were housed in individual wire-mesh cages with continuous access to water in a colony room with a 14-h-on/10-h-off light cycle. Food deprivation was maintained by allowing $90-$ min access to free food in their home cages after the termination of experimental sessions. All subjects had had prior experience in a study of leverpress acquisition under delayed reinforcement contingencies, conducted in a different apparatus (see Williams, Preston, \& de Kervor, 1990, Experiment 3, for details).

\section{Apparatus}

Two identical custom-built conditioning chambers, $24 \mathrm{~cm}$ wide $X$ $20 \mathrm{~cm}$ high $\times 26 \mathrm{~cm}$ long, were contained within a larger soundinsulating shell, which was equipped with an electric fan for ventilation. The interior chamber was constructed of Plexiglas except for a sheet-metal rear wall and wire-grid floor. The front panel of the chamber was painted black; the remaining walls and ceiling were clear Plexiglas. Mounted on the front panel $11.5 \mathrm{~cm}$ above the floor were two nonretractable stainless steel levers, $3 \mathrm{~cm}$ in width and protruding $1.8 \mathrm{~cm}$ into the chamber. Each lever required a force of at least $0.2 \mathrm{~N}$ for operation, with the only feedback of a response being the action of a microswitch connected to the other end of the lever. Directly below each lever and $2 \mathrm{~cm}$ above the grid floor was a pellet chute into which either standard 45-mg Noyes chow pellets (improved formula A) or $45-\mathrm{mg}$ sucrose pellets (Noyes formula F could be delivered. Sucrose pellets were delivered from a $28-\mathrm{V}$ pellet dispenser (Gerbrands Model G5100) located directly behind the left lever; chow pellets were delivered from an identical pellet dispenser located behind the right lever. Mounted $4 \mathrm{~cm}$ above the outer edge of each lever was a $28-\mathrm{V}$ miniature light (Sylvania 28 ESB bulb) encased within a recessed bulb holder. Located on the outside of the left side wall was a clicker module (Coulbourn Model E12-05), which presented a $4.5-\mathrm{Hz}$ auditory clicking stimulus. Mounted on the rear portion of the ceiling was a $4-\Omega$ speaker through which an $80-85 \mathrm{~dB}$ white noise could be delivered. Mounted on the outside of the right wall of the interior chamber was an unshielded 28-V lamp (bulb 1820), which could illuminate the entire experimental chamber with white light.

\section{Procedure}

In the first session of training, all subjects were trained to press the left lever on a continuous reinforcement schedule. The right lever was inoperative throughout the experiment. During this pretraining, only the white noise was present; the light and the clicker, which were later to serve as discriminative cues, were not presented. The white noise was present throughout the experimental sessions for the duration of the experiment. Training during this session occurred until 100 reinforcers had been obtained.

The general outline of the training procedure is shown in Table 1. For the blocking groups, Sessions 2-5 involved the introduction of the discriminative stimulus (A) used initially to establish stimulus control over the leverpress. During the first session, the discriminative stimulus (either the clicker or the houselight) was presented after an ITI determined by a variable time (VT) 1-min schedule. The duration of the initial presentation was $5 \mathrm{~min}$; this was then gradually reduced to $5 \mathrm{sec}$, which was the duration used for the remainder of training. During the stimulus, leverpresses were reinforced on a continuous reinforcement schedule. Responses in the absence of the discriminative stimulus were not 
reinforced. In addition, any responses during the 5-sec period prior to the scheduled trial period cancelled the trial. Training during this initial phase continued until all subjects within a squad (4 subjects) had reached a discrimination index of at leat $90 \%$ (i.e., $90 \%$ percent of all responses had to occur during the discriminative stimulus). Pretraining typically required $5-7$ sessions of training with the 5-sec trial period. Subjects within a given squad always received the same number of training sessions.

Compound-stimulus training began after discriminative control by element A had been established. Training during the compoundstimulus phase was identical to that during pretraining except that the second stimulus element (B) was added. Training with the compound stimulus continued for a minimum of eight sessions and until all control subjects within the squad had attained the $90 \%$ discrimination criterion.

Control subjects were given a second day of pretraining with the continuous reinforcement schedule with the discriminative stimuli absent, and then were begun on training with the compound stimulus. Sessions $2-5$ of this training involved the same gradual shortening of the trial period as was used for the blocking subjects during initial training with the pretrained element alone.

Testing for stimulus control continued for three sessions and were the same for all subjects. The first 2 test days consisted of presentations of stimulus B, which was added during the compoundstimulus training of the blocking subjects, with the same discrimination contingencies as were used during training (i.e., continuous reinforcement during the signal, and extinction in its absence). The third test session consisted of the presentation of stimulus $\mathrm{A}$, which had been used as the pretrained element for the blocking subjects.

The different subjects were divided into three groups: control subjects, which received no pretraining prior to training with the compound stimulus; the blocking-different group, which received pretraining with one reinforcer and then compound-stimulus training with the other reinforcer; and the blocking-same group, which received both pretraining and compound-stimulus training with the same reinforcer. Subjects in all three groups that served in the same squad always received the same reinforcer during compound-stimulus training. Thirty subjects served in the control group, and 17 subjects served in each of the two blocking groups.

Different combinations of stimulus elements and reinforcers were used for different sets of subjects. For each blocking condition, 9 of the 17 subjects in each group received the sucrose pellets as the reinforcer during compound-stimulus training, while the remaining 8 of 17 subjects received the chow pellet during compound-stimulus training. Similar proportions of the 30 control subjects received the different reinforcers. For the subjects that received sucrose as the reinforcer during compound-stimulus training, 6 experienced the houselight as the pretrained stimulus and 3 experienced the clicker; for the subjects that received the Noyes pellet reinforcer during compound training, 4 experienced the houselight as the pretrained stimulus and 4 experienced the clicker. A similar distribution was used for the subjects in the control group.

For all subjects, during the first two sessions of training, five pellets of the reinforcer not used during the training were placed in the pellet chute prior to the experimental session. This preexposure to the alternative reinforcer was presented in order to reduce any neophobia effects when the reinforcer was made contingent on the discriminated operant later in training.

\section{Results}

By the last session of pretraining with stimulus A alone, all subjects in the two blocking groups were responding primarily during the discriminated trial periods. For subjects in the blocking-same group, the percentage of total responses that occurred during the discriminative stimulus was $93.2 \%$; for subjects in the blocking-different group, the corresponding percentage was $94.6 \%$. This difference did not approach statistical significance $(F<1)$. The mean response rates during the discriminative stimulus for the two groups were 16.2 responses/min for the blocking-same group and 19.0 responses/min for the blocking-different group. This difference also was not statistically significant $[F(1,32)=1.39$; for this and all subsequent statistical tests, the significance level used was .05].

By the last session of the compound-conditioning phase with the compound stimulus $\mathrm{A}+\mathrm{B}$, subjects in all groups were again responding primarily during the trial periods. The percentage of total responses to the stimulus compound was $91.9,94.6$, and 91.0 for the control, blockingsame, and blocking-different groups, respectively. These differences were not statistically significant $(F<1)$. With respect to the response rates during the compound stimulus, the differences were somewhat greater: $20.5,18.0$, and $14.0 \mathrm{responses} / \mathrm{min}$ for the control, blocking-same, and blocking-different groups. However, this effect was not statistically significant $[F(2,61)=2.08]$.

The data from the first session of testing with element B alone are shown in Figure 1 in terms of three measures: the discrimination index to element B (percent of total responses occurring in the presence of element B), absolute response rate to element $\mathrm{B}$, and the normalized response rate to element $\mathrm{B}$. This last measure was calculated by dividing the absolute response rate to element $\mathrm{B}$ by the absolute rate to the $\mathrm{A}+\mathrm{B}$ compound during the last session of compound-discrimination training. Response rates were normalized because of the considerable variability across subjects in absolute response rates that was evident from very early in training.

The top portion of Figure 1 shows the effect of the blocking procedure on the accuracy of stimulus control by element $\mathrm{B}$. The mean proportions of correct responses were $.86, .77$, and .71 for the control, blocking-same, and blocking-different groups, respectively. This difference was subjected to a three-way analysis of variance (ANOVA) in which the factors were the pretraining condition (the group conditions shown in Figure 1), the reinforcer (sucrose vs. chow pellets) that was presented in the compound conditioning phase, and the stimulus that served as stimulus B (the clicker vs. the houselight). The effect of pretraining was significant $[F(2,52)=3.61]$, as was the effect of the stimulus serving as element $\mathrm{B}[F(1,52)=8.91]$, with greater discrimination accuracy occurring when the houselight served as stimulus B than when the clicker served as stimulus B (.85 vs. .72). Also significant was the interaction between the type of reinforcer and the stimulus $[F(1,52)=5.80]$. This probably reflected the spatial arrangement in the chamber, with the clicker and sucrose feeder located on the left side of the chamber and the houselight and chow-pellet feeder located on the right side of the chamber. Individual means for the four combinations of stimulus and reinforcer were $.87, .82$, .80 , and .63 for the houselight-chow, houselight-sucrose, click-sucrose, and click-chow combinations, re- 

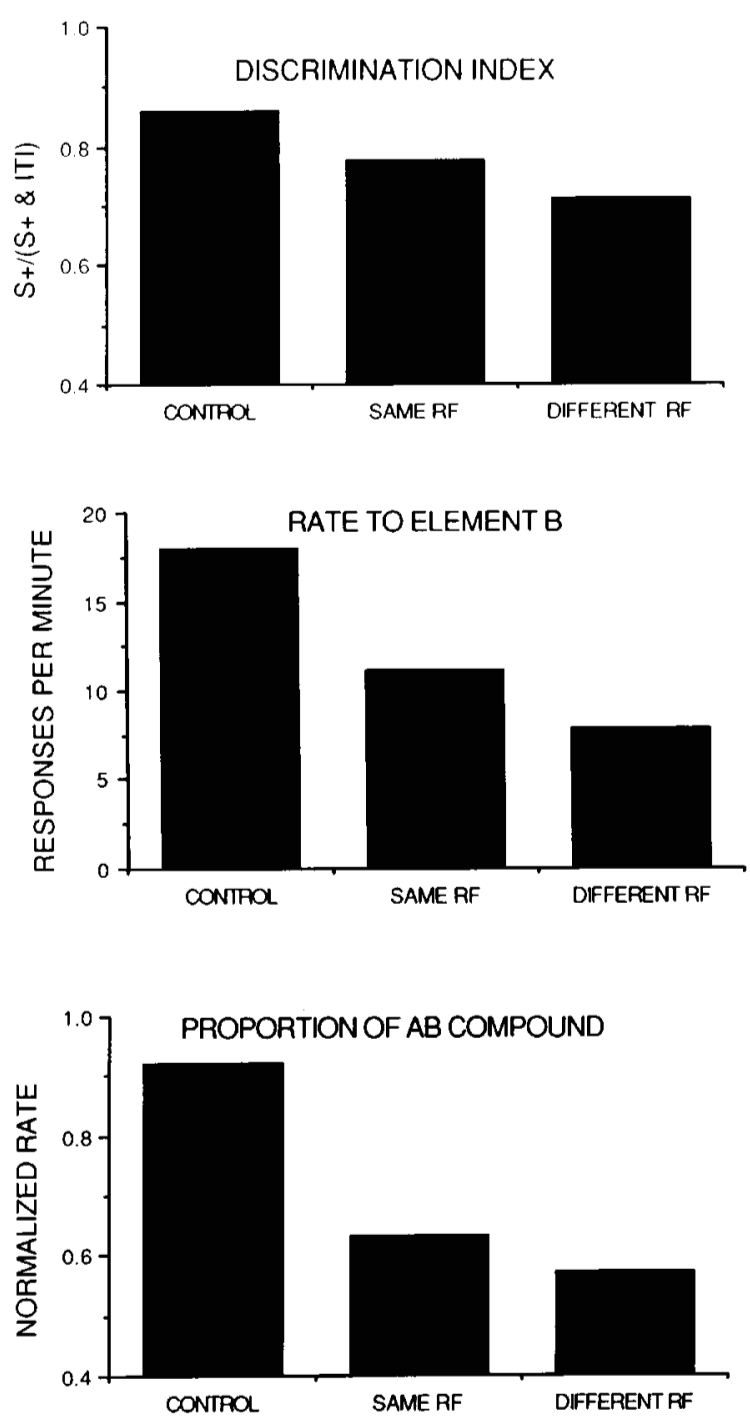

Figure 1. Results from the first test session in Experiment 1 with element $B$ alone. The top panel shows the percentage of total responses that occurred in the presence of stimulus $B$. The middle panel shows the absolute response rates in the presence of stimulus $B$. The bottom panel shows the normalized response rates to stimulus $B$, using, as the reference base, the response rates during the last session of training with the $A+B$ compound.

spectively. All other effects of the ANOVA were nonsignificant.

Comparisons between individual pretraining conditions were made with a Newman-Keuls test using a .05 confidence level. The result was that the only significant difference was that between the control and blockingdifferent conditions.

The relatively small effect of the pretraining variable on the discrimination index occurred because of the manner in which the blocking effects were primarily manifested. When testing occurred to element B alone for subjects in either blocking group, the primary change from the compound conditioning phase was in terms of absolute response rate during the discriminative stimu- lus, while little change occurred with respect to responding during the ITI. Response rate during the ITI was typically very low, as it had been during the compound conditioning phase, which meant that even large changes in response rate during the discriminative stimulus would have little impact on the discrimination index.

The middle portion of Figure 1 shows the absolute response rates to element $\mathrm{B}$ during the first test session. The mean values were $18.0,11.1$, and 7.8 responses $/ \mathrm{min}$ for the control, blocking-same, and blocking-different conditions, respectively. Again, a three-way ANOVA was conducted, with the result that only the effect of the pretraining variable was significant $[F(2,52)=7.71]$. Individual comparisons with the Newman-Keuls tests showed that the means of both blocking conditions were significantly less than the mean value of the control condition, and that the difference between the two blocking conditions was not significant.

The bottom portion of Figure 1 shows the results for response rate to element $B$ normalized for each subject relative to the response rate that occurred to the $A+B$ compound during the last day of compound-discrimination training. Here the averages over subjects were .92, .63, and .57 for the control, blocking-same, and blockingdifferent conditions, respectively. The three-way ANOVA showed that only the effect of groups was significant $[F(2,52)=8.12]$. Individual comparisons with the Newman-Keuls tests again showed that the mean values of both blocking conditions were significantly less than the mean values of the control condition, and that the difference between the two blocking conditions was not significant.

Results from the second session of testing with element B alone are not reported because the effects were weaker and more variable across subjects. This was due to the subjects' improving their discrimination performance to element B over the course of testing with the reinforcement contingencies remaining in operation.

After the two sessions of testing with element B alone, a third session of testing was presented with element $\mathrm{A}$ alone. In terms of the discrimination index, the mean proportion of total responses to the discriminative stimulus were $.85, .90$, and .88 for the control, blocking-same, and blocking-different groups, respectively. A three-way ANOVA failed to find any significant effects ( $F<1$ for the main effect of pretraining). Similar results were obtained for the absolute response-rate measure, and the mean rates were 18.8 , 17.6 , and 16.3 responses/min for the control, blocking-same, and blocking-different conditions. A threeway ANOVA again failed to find a significant effect of pretraining $(F<1)$, but here the effect of the stimulus serving as element A was significant $[F(1,51)=5.05]$. The mean response rates were 20.6 for the houselight and 14.5 for the clicker.

The failure to find a significant difference between the two blocking conditions occurred despite there being clear evidence that the change in reinforcers was discriminated by the blocking-different subjects. This was 
seen on the first session of compound-stimulus training after element $\mathrm{B}$ was added, during which response rates were substantially disrupted by the change in the reinforcer. Figure 2 shows this effect in terms of the response rate to the $\mathrm{A}+\mathrm{B}$ compound relative to the response rate during the last session of pretraining with element A alone. Four separate conditions are shown: Subjects in the blocking-same conditions are subdivided according to the reinforcer used throughout, and subjects in the blocking-different conditions are subdivided according to the direction of the switch in reinforcers. Some disruption occurred even with the blocking-same subjects, as the mean proportion of the pretraining rate was .73 and .79 for those continued on the sucrose and chow pellets, respectively. The disruption for these subjects was presumably due to the addition of the new stimulus element B, and thus represents a form of external inhibition. The disruption for the subjects with the switch in reinforcers was much greater, as the mean normalized rates were .47 for the subjects switched from chow pellets to sucrose and .45 for those switched in the opposite direction.

Because the effect of shift in reinforcer produced similar effects regardless of the particular reinforcer initially presented, the data shown in Figure 2 were analyzed with a simple one-way ANOVA to compare subjects continued on the same reinforcer with those for which the reinforcer was switched. The difference was statistically reliable $[F(1,32)=20.7]$.

\section{Discussion}

The results are consistent with those of Ganesan and Pearce (1988) in showing that blocking was not attenuated by a change in the reinforcer between the pretraining with element $\mathrm{A}$ alone and the compound condition-

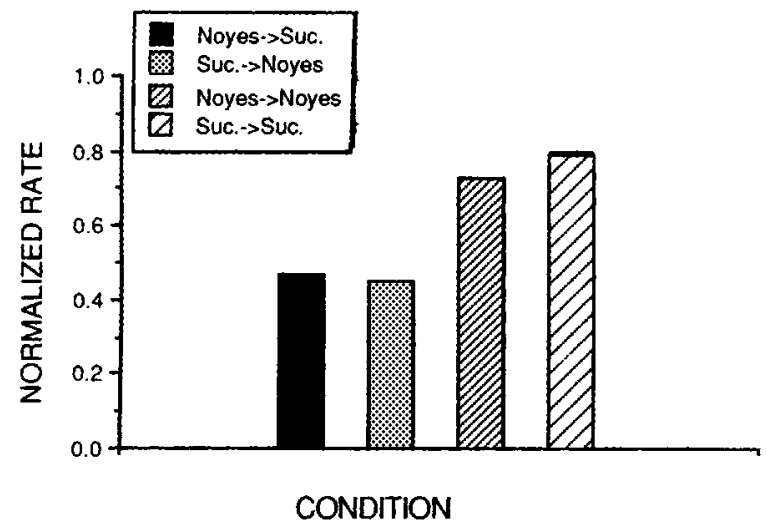

Figure 2. Results from the first session of training with the $A+B$ compound for four different blocking conditions. The first two bars show results from the blocking-different group subdivided according to the direction of the change in reinforcers. The last two bars show the results from the blocking-same group subdivided according to which reinforcer was used throughout training. The normalized rate measure was calculated by dividing the rate to the $A+B$ compound by the rate during the last session of training with element $A$ alone. ing phase with the compound $\mathrm{A}+\mathrm{B}$. The failure to find a significant reduction in blocking with the blockingdifferent group cannot be attributed to a lack of sensitivity for the various measures of stimulus control because all were in the opposite direction from that expected. That is, what difference there was between the blocking-same and blocking-different conditions showed greater blocking in the blocking-different condition. The results thus establish that the failure of unblocking to occur as a function of changing the identity of the reinforcer is not restricted to Pavlovian procedures, but occurs in operant procedures as well.

It is also noteworthy that the blocking effect in the blocking-different condition occurred despite clear evidence that discrimination of the change in reinforcer had occurred. As shown by the response rates during the first day of compound $\mathrm{A}+\mathrm{B}$ training, subjects with the changed reinforcer responded at a substantially lower rate. Ganesan and Pearce (1988) reported a similar difference in their Experiment 4, in which the location of the reinforcer/conditioned response (both were defined by the location of the reinforcer magazine) was changed between pretraining and compound conditioning, but showed no effect of the change in reinforcers when the location of reinforcer stayed the same (Experiments 1-3). The significance of the disruption of responding that occurred on the first day of compound conditioning is uncertain, in part because some degree of disruption occurred for the subjects without a change in reinforcer simply because of the change from the single pretrained stimulus to the compound stimulus. How such "external inhibition" effects affect the degree of blocking cannot be determined from the present comparison between the blocking and the control subjects, which allows only the conclusion that some degree of blocking occurred, which may or may not have been attenuated by the decrease in stimulus control that was evident on the first day of compound-stimulus training. Nevertheless, it is clear that the additional amount of "external inhibition" caused by the change in reinforcers did not increase the degree of unblocking that occurred.

Although the present results are consistent with Ganesan and Pearce's (1988), they are in conflict with those of conceptually similar studies by Colwill and Rescorla (1990, Experiment 3) and Rescorla (1990, Experiment 1). In the pretraining phase of their studies, each of two responses led to one of two different reinforcers in the presence of a discriminative stimulus (A), while neither reinforcer was available during the absence of the stimulus. In the compound conditioning phase, two new stimuli were added to form two separate stimulus compounds, BA and CA. In the presence of BA, the responsereinforcer relations were the same as those used during pretraining, while in the presence of CA, the opposite response-reinforcer relations were in effect. Then elements $\mathrm{B}$ and $\mathrm{C}$ were tested alone to determine if they had acquired stimulus control over responding. In both experiments, element B failed to acquire stimulus control, indicating blocking, while element $\mathrm{C}$ did acquire con- 
trol. The latter finding demonstrates that changing the reinforcer that follows a response is apparently sufficient to prevent blocking from occurring, a finding in conflict with that reported here.

The present results are also in conflict with those of Holland (1988), who investigated the causes of "unblocking" in a Pavlovian procedure. In some conditions, either one or two reinforcers in series followed the conditioned stimulus (CS), and the nature of these two reinforcers was varied between the pretraining and compound conditioning phases. The pattern of results was complex, but the major finding relevant to the present study was that a deleted reinforcer (e.g., CS-A $\rightarrow$ RF$(\mathrm{X}+\mathrm{RF}-\mathrm{Y})$ in Phase 1 ; CS- $(\mathrm{A}+\mathrm{B}) \rightarrow \mathrm{RF}-\mathrm{X}$ in Phase 2$)$ caused blocking of stimulus $\mathrm{B}$ to be attenuated, although this unblocking effect could be masked by inhibitory associations under certain conditions. Despite their complexity, Holland's results provide strong support for the view that changes in the identity of the reinforcer may prevent blocking from occurring.

The reasons for the conflict between the various results that have been described are not clear. Ganesan and Pearce (1988) suggested that Konorski's (1967) distinction between the hedonic versus sensory representations of a reinforcer might be helpful in that blocking would be expected to occur as long as the change in reinforcers maintained the hedonic value of the original reinforcer even while the sensory characteristics were substantially altered. While such a notion is consistent with the present findings, it cannot explain those of Colwill and Rescorla (1990) and Rescorla (1990), who both used reinforcers of the same roughly equal hedonic value (Noyes chow pellets and sucrose solution).

An alternative possibility is the distinction between between-subject and within-subject designs. Withinsubject designs were used by Colwill and Rescorla, while between-subject designs were used in the present study and in that of Ganesan and Pearce (1988). It is possible that intermixing the different reinforcement relations within the same session may sensitize subjects to the particular characteristics of the reinforcer, and thus cause the different identities to be discriminated more accurately. Some evidence for the role of enhanced discrimination comes from Colwill and Rescorla (1990), who reported a significant blocking effect when two stimuli, one signaling the same response-reinforcer relationship as during pretraining and the second signaling the reverse relationship, were interspersed, but failed to find a significant effect when the training on the different relationships was presented in separate phases of training.

\section{EXPERIMENT 2}

This experiment provides a conceptual replication of Experiment 1, but with a within-subject design, as outlined in Table 2. The issue was whether the change in reinforcer identity between the single-stimulus pretraining and the compound-stimulus training would have an ef-
Table 2

Outline of Procedure for Experiment 2

\begin{tabular}{|c|c|c|c|}
\hline Group & Phase 1 & Phase 2 & Test \\
\hline \multicolumn{4}{|l|}{ Blocking } \\
\hline Sucrose & $\begin{array}{l}\mathrm{L}(\text { left)-sucrose } \\
\mathrm{L} \text { (right)-ext }\end{array}$ & $\begin{array}{l}\text { L(left)-sucrose } \\
(\mathrm{L}+\mathrm{A}) \text {-sucrose } \\
(\mathrm{L}+\mathrm{B}) \text {-chow }\end{array}$ & A, B, L \\
\hline Chow & $\begin{array}{l}\text { L(left)-chow } \\
\text { L(right)-ext }\end{array}$ & $\begin{array}{l}\mathrm{L}(\text { left }) \text {-chow } \\
(\mathrm{L}+\mathrm{A}) \text {-chow } \\
(\mathrm{L}+\mathrm{B}) \text {-sucrose }\end{array}$ & A, B, L \\
\hline \multicolumn{4}{|l|}{ Control } \\
\hline Sucrose & $\begin{array}{l}\text { L(right)-sucrose } \\
\text { L(left)-ext }\end{array}$ & $\begin{array}{l}\mathrm{L}(\text { left)-sucrose } \\
(\mathrm{L}+\mathrm{B}) \text {-chow } \\
(\mathrm{L}+\mathrm{A}) \text {-sucrose }\end{array}$ & A, B, L \\
\hline Chow & $\begin{array}{l}\mathrm{L} \text { (right)-chow } \\
\mathrm{L}(\text { left)-ext }\end{array}$ & $\begin{array}{l}\mathrm{L}(\mathrm{left}) \text {-chow } \\
(\mathrm{L}+\mathrm{A}) \text {-chow } \\
(\mathrm{L}+\mathrm{B}) \text {-sucrose }\end{array}$ & A, B, L \\
\hline
\end{tabular}

Note-The stimuli serving as A and B (a click or tone) was counterbalanced across subjects within a group.

fect on the occurrence of blocking with the withinsubject design that differed from that in Experiment 1, which used a between-subject design.

\section{Method}

\section{Subjects}

Twenty-four albino rats with experimental histories similar to those in Experiment 1 were maintained in the same manner as described for Experiment 1.

\section{Apparatus}

The same apparatus was again used, with the modification that a Radio Shack tone generator was added. This emitted a tone from the overhead speakers of complex-wave form with a major frequency of $845 \mathrm{~Hz}$ and an intensity of approximately $84 \mathrm{~dB}$ SPL.

\section{Procedure}

During the first two sessions, the subjects were trained to press the left lever in the absence of any discriminative stimulus until 200 reinforced leverpresses had occurred. During this time, 10 5 -sec presentations of both the tone and the clicker occurred, during which the leverpress contingencies were unchanged. Unlike Experiment 1, the white noise was not presented; masking sound was provided only by the ventilating fans in the outer insulating shell containing the conditioning chambers.

Pretraining. After vigorous leverpressing was established for all subjects, the pretraining phase, involving a discrimination between the left and right panel lights, was begun. For 16 subjects (blocking group), the left panel light served as the $\mathrm{S}+$ and the right panel light was the $\mathrm{S}-$. For 8 subjects (control group), these contingencies were reversed. One or the other light was presented for 5 -sec trial periods separated by a variable ITI, which was VT $10 \mathrm{sec}$ initially and then gradually increased to VT $60 \mathrm{sec}$. During the last six pretraining sessions, the VT schedule determining the ITI was alternated over sessions between VT $30 \mathrm{sec}$ and VT $60 \mathrm{sec}$. When the $\mathrm{S}+$ light was presented, a single reinforcer was presented at the end of the $5-\mathrm{sec}$ trial period if at least one leverpress had occurred during the trial. During the S- light, leverpressing had no consequences, as was the case for leverpresses during the ITIs in the absence of the S+. Training continued with these contingencies for 18 sessions, with each session continuing for 100 trials in which $\mathrm{S}+$ and $\mathrm{S}-$ trials were randomly alternated.

For both groups, half of the subjects were trained with the 45-mg sucrose pellet as the reinforcer and half were trained with the $45-\mathrm{mg}$ Noyes Formula A chow pellet as the reinforcer. The sucrose pellet 
was presented from the feeder on the right side of the front panel; the Noyes chow pellet was presented from the feeder on the left side, and thus was directly below the left lever.

Compound stimulus training. During this phase, three different types of S+ trials were presented, those with the left panel light alone, those with the left panel light in compound with the clicker, and those with the left panel light in compound with the tone. For all of these, reinforcers were delivered at the end of the 5-sec trial period if at least one leverpress had occurred during the trial. No $\mathrm{S}-$ trials were presented. One-half of the trials during the session were to the light alone; the remaining half were equally divided between the two compound stimuli. Note that, during pretraining, the left light had served as the $\mathrm{S}+$ for the subjects in the blocking group but as the $\mathrm{S}-$ for the control subjects.

For both groups, one of the compound stimuli was paired with the sucrose reinforcer and one was paired with the chow pellet. The auditory stimulus (the clicker or tone) paired with the different reinforcers was counterbalanced over subjects for both groups. Thus, one target stimulus was compounded with the light and paired with the same reinforcer as that with the light alone, and one target stimulus was compounded with the light and paired with a reinforcer different from that with the light alone. Training during the compound-stimulus phase continued for eight sessions, with each session consisting of 100 trials.

Test phase. The first test session with the tone and clicker elements presented alone occurred with extinction in effect. For half of the subjects, only the tone and clicker were presented. There were no light-alone presentations, but trials during which the light alone would have been presented were recorded in the same manner as if the light had been there to provide an index of the baseline response rate in the absence of any discriminative stimulus. For the remaining half of the subjects, the tone-alone and clickeralone presentations were interspersed with light-alone trials; extinction was in effect for all three types of trials. For both conditions, the clicker was presented for 25 presentations and the tone was presented for 25 presentations. During the second test session, all subjects received light-alone, clicker-alone, and tone-alone trials, interspersed, and the reinforcer contingencies were reinstated. For the clicker-alone and tone-alone trials, the reinforcer presented was the same as that paired with those stimuli during the compound training phase. Fifty light-alone trials and 25 clicker-alone and tone-alone trials were presented.

\section{Results}

\section{Pretraining}

The discrimination between the two panel lights was gradually acquired by all subjects. During the last 2 days of pretraining, the mean discrimination index (proportion of total trials with at least one response occurring during the $\mathrm{S}+$ ) was .89 for the subjects in the blocking group and .81 for subjects in the control group. This difference, while small, was statistically reliable $[F(1,22)=$ 4.29]. The apparent reason for the difference is that the spatial separation between the $\mathrm{S}+$ and the lever was greater for the control subjects than for the blocking subjects: both responded to the left lever, while the right light was the $\mathrm{S}+$ for the controls and the left light was the $\mathrm{S}+$ for the blocking subjects. There was no difference for either group in the discrimination index as a function of which reward served as the reinforcer.

The proportion of trials in which at least one response occurred during presentations of the left light was .92 for the blocking subjects. The corresponding proportion for responses to the right light was .87 for the control subjects, while their proportion for responses to the left light, their $\mathrm{S}-$, which was later paired with the auditory stimulus during the compound-stimulus training, was .22. There was no overlap between the control and blocking subjects in responding to the left light, showing that the pretraining had established different associative strengths to that stimulus for the two groups.

\section{Compound-Stimulus Training}

Despite the prior presentations of the tone and clicker, their presentations during the first compound-stimulus session substantially disrupted behavior, with substantially greater disruption caused by the clicker than by the tone. For the blocking subjects, the proportion of trials with a response to the panel light presented alone was .86 , while the proportion of trials with a response to the light + tone was .80 and that to the compound of the light + clicker was .48. A similar pattern was seen for the control subjects, which received food paired with the left panel light for the first time. Their corresponding percentages were .62 for the light alone, .56 for the light plus tone, and .30 for the light plus clicker.

Performance during the first compound conditioning session was relatively little affected by whether the reinforcer for the compound-stimulus trials was the same as or different from that to the light alone. For the blocking

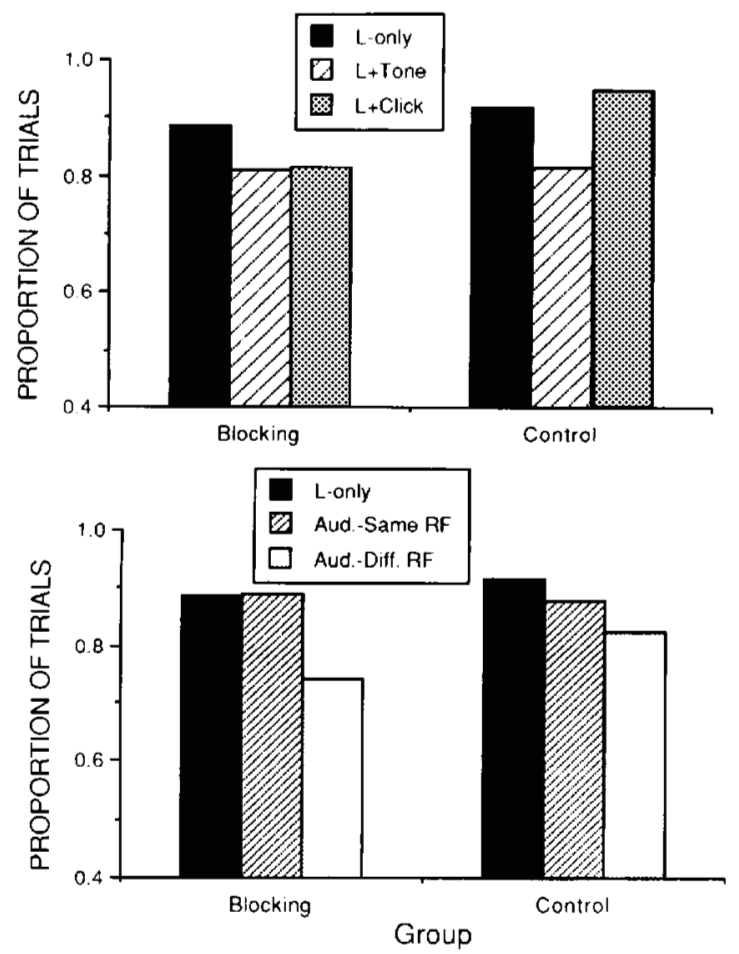

Figure 3. Results from the last two sessions of compound discrimination training of Experiment 2. The top panel shows the results subdivided according to the physical identity of the auditory element; the bottom panel shows the results subdivided according to which compound was paired with the reinforcer that was the same as, or different from, that with the light element alone. 
group, the proportion of trials with a response was .65 when the reinforcer was the same and .63 when the reinforcer was different. For the control subjects, the corresponding proportions were .46 and .41 . Neither difference approached statistical reliability.

Continued training with the compound stimulus produced a substantially different pattern of results. The results during the last two sessions of compound training are shown in Figure 3. In the top panel are the results subdivided according to the physical identity of the auditory stimulus. For the blocking subjects, there was no difference as a function of whether the added element was the tone or clicker, as the proportion of trials with a response was .81 for both stimulus compounds, slightly below the .88 value when the light was presented alone. For the control subjects, a nonsignificant difference did occur between the tone and clicker (.81 vs. .94, as opposed to the .91 for the light alone), but this difference was in the opposite direction from that obtained during the first session of compoundstimulus training.

In the bottom portion of Figure 3, the results are subdivided according to whether the reinforcer following the compound-stimulus trials was the same as or different from that following the light alone. For the blocking subjects, the proportion of trials with a response when the same reinforcer occurred was similar to that of the light-alone trials (.89 vs. .88), whereas the proportion when the reinforcer was different was reduced (.74 vs. .88). The difference between the same and different reinforcer was statistically reliable $[t(15)=3.56]$. For the control subjects, a similar difference occurred, but was smaller in magnitude and not statistically reliable.

\section{Test Results}

The overall blocking effect is displayed in Figure 4, which shows the results of the two different test sessions in which the auditory stimuli were presented alone. The results are averaged over both the physical identity of the auditory stimulus and whether the stimulus was paired with a reinforcer that was the same as or different from

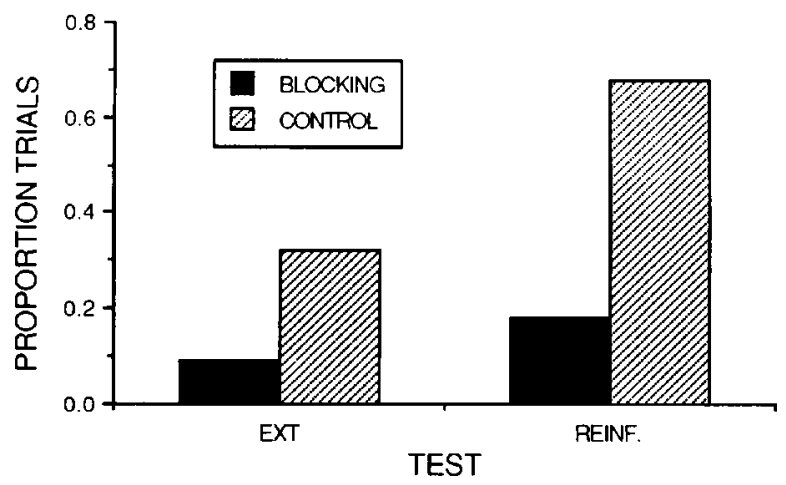

Figure 4. Control by the added auditory element for the blocking and control groups, presented separately for the two types of test session.
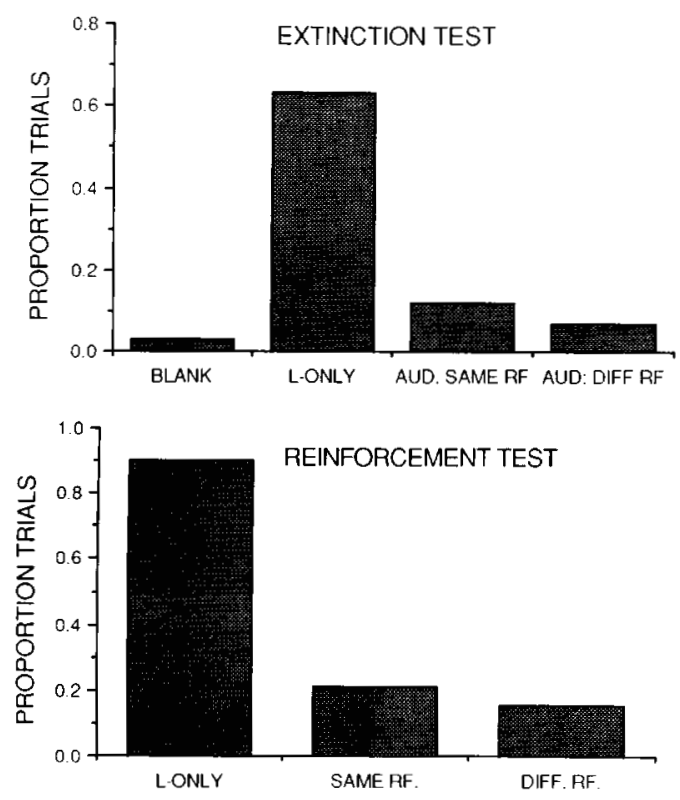

Figure 5. Results from the blocking subjects subdivided according to which auditory element was paired with the reinforcer that was the same as, or different from, that with the light alone. The top panel shows the results from the extinction test; the bottom panel shows the results from the reinforcement test.

that of the light alone. It is evident that substantially greater responding occurred for the control subjects, for both test sessions. For the extinction test, the mean proportion of trials with a response to the auditory stimulus alone was .32 for the control subjects and .09 for the blocking subjects, and this difference was statistically reliable $[F(1,22)=24.42]$. For the reinforcement test, the corresponding proportions were .68 and .18 , and this difference was also reliable $[F(1,22)=47.60]$. Thus, the prior training with the left panel light as $\mathrm{S}+$ did produce a significant attenuation of learning about the new stimulus element.

Given that a significant blocking effect occurred, the issue is whether it varied as a function of whether the reinforcer paired with the added auditory element was the same as or different from that paired with the light during pretraining. Figure 5 shows the results during the test sessions: the top portion shows the results from the extinction test; the bottom portion shows the results for the test when the reinforcement contingencies were reinstated. For purposes of comparison, the percentage of trials with a response are also shown for the blank trials (for the EXT test only), in which no stimulus was present, and for when the light was presented alone. It is evident that the auditory stimuli exerted little control over behavior, and that the degree of responding was slightly less when the reinforcer had been different from that paired with the light alone. For the EXT test, the proportion of trials with a response was .12 for the same reinforcer stimulus and .07 for the different reinforcer stimulus. For the reinforcement test, the corresponding percentages were .21 and .16. For purposes of statistical 
analysis, a two-way ANOVA was conducted with the physical nature of the reinforcer (sucrose vs. chow pellet) as one factor and the relation of the reinforcer to the light-alone reinforcer (same vs. different) as the second factor. All other variables were excluded because preliminary analysis had shown that they had no effect. The effect of sucrose versus chow approached significance $[F(1,14)=4.34, p=.06]$, while the effect of the samedifferent variable was significant $[F(1,14)=4.74]$. A corresponding analysis for the reinforcement test failed to produce a significant effect for either variable [for sucrose vs. chow, $F(1,14)<1$; for same vs. different, $F(1,14)=2.87]$.

\section{Discussion}

The results were generally similar to those of Experiment 1 in that "unblocking" did not occur to the added element of the stimulus compound paired with a reinforcer different from that paired with the pretrained stimulus element alone. Instead, slightly less responding occurred to the element paired with the different reinforcer as the pretrained element than it did to the element paired with the same reinforcer. A similar difference was noted in Experiment 1 with the between-subject design, with the only difference in outcome being that, here in Experiment 2, this difference was statistically significant. The results are the opposite of what would be expected on the basis of the previous work of Rescorla (1990).

The greater blocking that occurred to the stimulus paired with the different reinforcer appears to be partly due to the pattern of behavior that developed during the compound-stimulus training prior to the test phase. As shown in Figure 3, the rate of responding to the compound paired with the different reinforcer was significantly below that to the compound paired with the same reinforcer, so that it appears that the pattern of results obtained during the test sessions was a carryover from the pattern established during training. Unfortunately, the pattern established during compound training is very difficult to understand in its own right. The rate of responding to the compound stimulus was presumably the result of the combined associative strengths of each element of the compound. If unblocking occurred because the different reinforcer was used, this would imply that the auditory element paired with the different reinforcer should have some degree of associative strength from its pairing with the new reinforcer, which would be combined with the associative strength from the light element, thus producing an increased rate of responding during the compound conditioning phase. Instead, response rate was reduced, suggesting that the added stimulus element controlled behavior that interfered with barpressing controlled by the pretrained light. Perhaps the use of different feeder locations for the two different reinforcers engendered a greater degree of "goal tracking" than occurred with a single feeder site. Such an effect would presumably reflect only a performance effect and not associative differences in the degree of stimulus control.

\section{EXPERIMENT 3}

Despite the use of a within-subject design in Experiment 2, its failure to reveal an unblocking effect leaves unresolved the nature of the conflict with the previous results of Colwill and Rescorla. Experiment 3 was thus intended to pursue further the nature of the discrepant findings. Experiment 2 differed from the Colwill and Rescorla (1990) and Rescorla (1990) experiments in two notable ways: (1) In Experiment 2, we employed a single response, whereas they used two different responses; and (2) in Experiment 2, we used trial durations much shorter than theirs ( 5 vs. $30 \mathrm{sec}$ ). It is possible that the function of the present stimuli was affected by either or both of these variables, in that both may have caused the Pavlovian CS+ properties to be more important at the expense of their functions as operant discriminative stimuli. Consequently, in Experiment 3, we employed two separate responses in the presence of the different stimuli, and the trial period was extended from 5 to $30 \mathrm{sec}$.

\section{Method}

Thirteen male albino rats with similar histories to those trained in Experiments 1-2 were maintained as described for Experiment 1 . The same chambers were also used. The only difference was that here both response levers were used instead of only the left lever in Experiments 1 and 2.

Eight subjects were assigned to the blocking condition and 5 were assigned to the control condition. Training for the blocking subjects began with the panel light continuously available and a continuous reinforcement (CRF) schedule operating for both levers. Presses of the left lever produced a 45-mg Noyes chow pellet; presses of the right lever produced a $45-\mathrm{mg}$ sucrose pellet. Training on this procedure continued until approximately 100 reinforcers had been received for each of two sessions. Beginning on the third session, the panel light was illuminated for 30 -sec stimulus periods interspersed with an ITI in which the panel light was off and extinction was in effect for both levers. The length of the ITI was gradually increased to a VT $60 \mathrm{sec}$, and the schedule for leverpresses was extended from CRF to VI $30 \mathrm{sec}$. When a reinforcer was scheduled, a probability gate assigned it randomly to ei-

Table 3

Outline of the Procedure for Experiment 3

\begin{tabular}{|c|c|c|c|}
\hline Group & Phase 1 & Phase 2 & Test \\
\hline Blocking & $\begin{array}{l}\text { L:left-chow } \\
\text { :right-sucrose }\end{array}$ & $\begin{array}{c}\text { L:left-chow } \\
\text { :right-sucrose } \\
(\mathrm{L}+\mathrm{A}): \text { left-chow } \\
\text { :right-sucrose } \\
(\mathrm{L}+\mathrm{B}): \text { left-sucrose } \\
\text { :right-chow }\end{array}$ & A, B, L \\
\hline Control & No training & $\begin{array}{c}(\mathrm{L}+\mathrm{A}): \text { left-chow } \\
\text { :right-sucrose } \\
(\mathrm{L}+\mathrm{B}): \text { left-sucrose } \\
\text { :right-chow }\end{array}$ & A, B, L \\
\hline
\end{tabular}

Note-The stimulus elements serving as A or B (noise or clicker) were counterbalanced across subjects within each group. 
ther the right or left lever, and it was held until obtained by the next response to that lever. Thus, each lever was associated with a VI 60 -sec schedule. Two to five sessions were needed to stabilize all subjects on the final training values, after which training was continued for 10 additional sessions.

Compound stimulus training involved sessions in which 32 30sec trials were presented, separated by a VT 60 -sec ITI. One-half of these trials involved presentation of the panel light alone in which presses of the left lever produced chow pellets while presses of the right lever produced sucrose pellets. The remaining half of the trials were divided equally between compound stimulus presentations, the panel light either in combination with the clicker or in combination with white noise. For one of these compound stimuli, the contingencies were identical to those with the panel light alone; for the other compound stimulus, the contingencies were reversed, so that presses of the left lever produced sucrose pellets while presses of the right lever produced chow pellets. Which auditory stimulus was associated with the same or reversed contingencies was counterbalanced over subjects. Nine sessions with the compound-stimulus conditions were presented. An outline of the procedure is shown in Table 3.

Subjects in the control condition were not given pretraining with the light; they were begun with the stimulus compounds at the start of training, without separate presentations of the panel light alone. Thus, either of two trial types was presented for 30 -sec periods, light+clicker or light + noise. The schedule during both trial types was initially CRF for both responses, and was gradually increased over the first two sessions to VI $60 \mathrm{sec}$. The ITI separating trials was also increased during this time to VT $60 \mathrm{sec}$. To maintain comparability with the blocking condition, eight presentations of each compound stimulus were presented each session. Three subjects in the control group had left leverpresses reinforced by chow and right leverpresses reinforced by chow and right leverpresses reinforced by sucrose pellets during the light+noise coupound, and the reverse contingencies during the light+click compound; the remaining 2 subjects received the opposite contingencies. Training for the control subjects continued for a total of 10 sessions, the first 2 of which involved gradually increasing the reinforcement schedule and ITI value.

At the end of compound-stimulus training, all subjects received the identical test procedure. During the first test session, a single pellet of each type was placed in each pellet chute at the start of the session and extinction then remained in effect for the remainder of the session. Three types of trials were presented in each session, the panel light alone, the clicker alone, and the noise alone. For all subjects, 32 trials were presented, 16 with the light alone and 8 with each of the two auditory stimuli. Thus, the only change for the blocking subjects from the regular training procedure was that the light was removed from the compound involving the auditory elements. For the control subjects, in addition, the test session was the first occasion on which the light was presented alone.

On the second test session, the sequence of stimulus presentation remained the same, but reinforcement was now available in all components.

\section{Results}

For subjects in the blocking condition, pretraining with the light alone proceeded smoothly, with all subjects learning to emit more than $90 \%$ of their responses in the presence of the light. During the last two sessions of pretraining, the average rate of responding to the left lever, which produced chow pellets, was 14.5 responses/ min, while that to the right lever, which produced sucrose pellets, was 21.9. This preference was inconsistent across subjects and failed to reach significance using a paired $t$ test $[t(7)=1.72]$.

The introduction of the auditory stimuli on the first day of compound stimulus training disrupted behavior to the light alone only slightly, the rate to the left lever on the first session being 12.2 and that to the right lever, 19.8. The corresponding rates to the light + noise combination were 17.8 and 20.1, indicating a mild facilitation of behavior, perhaps due to stimulus intensity dynamism. The corresponding rates to the light + clicker combination were considerably lower, 10.9 and 13.4 to the left and right levers, respectively.

The response rates on the first compound session as a function of the reinforcer contingencies being the same as or different from that with the light alone, averaged over the two auditory stimuli and both levers, were 31.7 for the same contingencies and 30.5 for the different contingencies. Thus, on the first day of training, no disruption occurred due to the change in reinforcement contingencies per se.

A somewhat different pattern of results occurred during the last two sessions of compound stimulus training. During the light alone, the mean rates were 14.8 to the left lever and 20.9 to the right lever, indicating little change over the nine sessions of training. During the light + noise compound, the corresponding rates were 18.8 and 23.1 ; during the light+clicker, they were 19.0 and 19.9. Thus, unlike the first compoundstimulus session, here the rates to the noise and clicker were more similar, as the disruption caused by the clicker had largely disappeared by the end of training. The rates during the last two compound sessions as a function of the reinforcement contingencies, summed over both response levers, were 43.5 when the same reinforcer contingencies occurred during the compound, as they did during the light alone, and 37.0 when the contingencies were reversed. This difference was consistent across subjects and was statistically significant $[t(7)=3.13]$. Thus, having the contingencies during the stimulus compounds reversed from those during the light alone significantly disrupted the behavior by the end of training, although no such effect was evident on the first day of compound-stimulus training.

The subjects in the control group had somewhat lower response rates during the last two sessions of compoundstimulus training, probably because the reinforcement schedules were increased more abruptly for them than for the blocking subjects in order to ensure that the control subjects not receive more exposure to the auditory stimuli than the blocking subjects had. The average response rates, summed over both levers, were 28.2 during the light + noise compound and 24.1 during the light + clicker compound. Summed over levers and the two types of compound-stimulus trial, the response rate maintained by the chow pellet was 24.4 and that maintained by the sucrose pellet was 27.8 .

The critical data are the results from the test sessions with the auditory elements presented alone for the first 
time. By comparing the results for the control and blocking subjects for the light alone and those averaged over the two auditory elements independent of their reinforcer contingency, Figure 6 provides the data that assess whether an overall blocking effect occurred. The response rate to the light alone was substantially higher for the blocking subjects than for the controls, with no overlap between the two groups. In contrast, response rates to the auditory elements were substantially higher for the controls than for the blocking subjects. Summed over both response levers, the mean rates to the auditory elements were 12.8 for the control subjects and 3.7 for the blocking subjects. The same pattern occurred during the second test session with the reinforcer contingencies reinstated, where the average rates to the auditory elements were 13.9 for the blocking group and 27.4 for the control subjects. These differences were analyzed statistically with a two-factor ANOVA, with group assignment as a between-group factor and type of test as a within-subject factor. The contrast between the blocking and control subjects was significant $[F(1,11)=15.6]$, as was the type of test $[F(1,11)=31.8]$. The interaction was not significant $(F<1)$. Thus, the control subjects acquired significantly more stimulus control of the auditory elements than did the blocking subjects, and this was exhibited during both types of testing.

Since a blocking effect had occurred, the critical test was whether it was differential for the auditory element correlated with the same reinforcement contingencies as the light alone versus for the auditory element correlated with the reversed reinforcement contingencies. Figure 7 shows the results as a function of the reinforcement contingency, along with response rates during the ITI in the absence of any external stimuli. The response rates during both auditory elements were slightly higher during the extinction test than during the ITI, as the mean rates for the same contingency, different contingency, and ITI were $4.5,2.9$, and 1.2 , respectively. The lower rates during the ITI were consistent across subjects, but this does not mean that the increase in re-

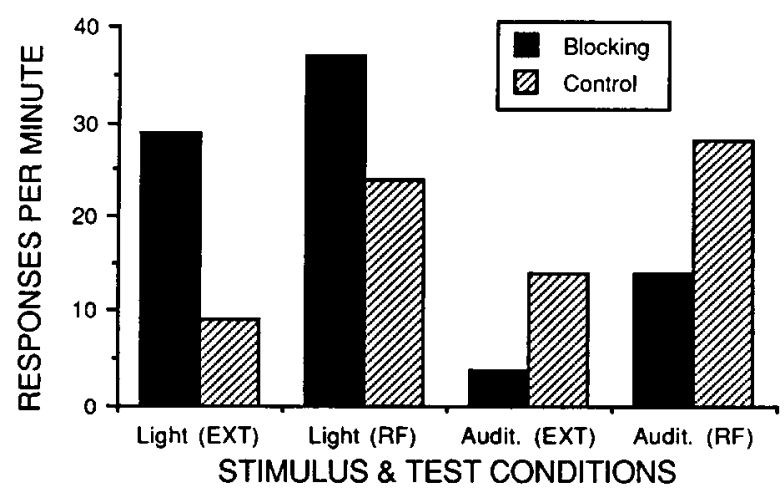

Figure 6. Response rates to the stimulus elements during the test sessions in Experiment 3, summed over both levers and separated for subjects in the blocking versus control conditions. Response rates in the presence of the two auditory stimuli are averaged together.

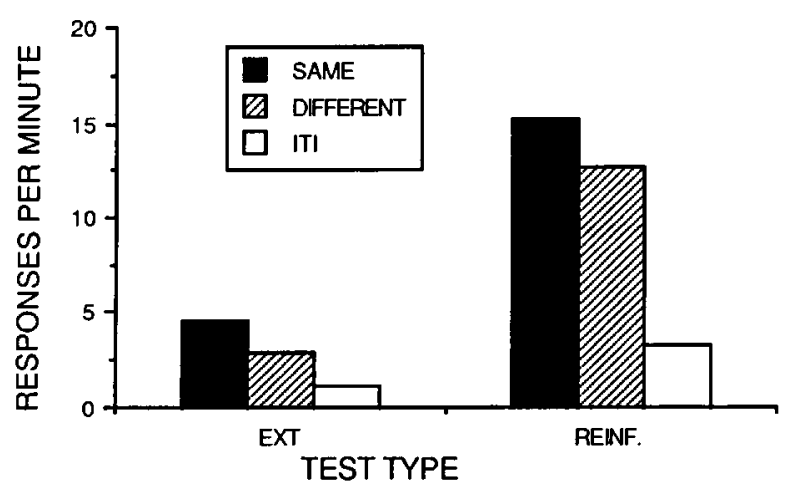

Figure 7. Response rates to the two auditory elements during the test sessions in Experiment 3, summed over both levers, as a function of whether the auditory element was paired with the same reinforcer contingencies as was the light or with different reinforcer contingencies. Also shown are response rates during the ITI in the absence of either auditory element.

sponding caused by the auditory stimuli was necessarily due to their associative history. It is possible, for example, that any type of significant stimulation would have produced a similar increase in responding. The more important comparison is that between the two auditory elements, and Figure 7 shows that the rates were higher to the auditory element correlated with the same reinforcement contingencies for both types of test. For the extinction test, this difference was significant, as assessed by a paired $t$ test $[t(7)=3.26]$. For the reinforcement test, however, the difference was not significant $[t(7)=.81]$. Thus, for neither test is there any evidence that more learning occurred to the stimulus associated with different reinforcement contingencies than to those correlated with the panel light with which the auditory elements were compounded.

\section{Discussion}

Despite the procedural changes from Experiment 2, the results were essentially similar. Blocking occurred regardless of whether the added stimulus in the compound-stimulus training phase was associated with a reinforcer that was the same as or different from that used in single-stimulus pretraining. Also as in Experiment 2, the difference that did occur was less stimulus control by the stimulus associated with the changed reinforcer contingencies than by the stimulus with the unchanged contingencies. Again, this appears to be attributable to the fact that the changed reinforcer contingencies supported a lower response rate at the end of compound-stimulus training.

The absence of any evidence of unblocking in the present study is surprising given that the design of Experiment 3 was essentially similar to that used by Rescorla (1990) and Colwill and Rescorla (1990). The major procedural difference between their studies and the present one is the nature of the reinforcer that was used: They used standard chow pellets versus sucrose solution, while we used chow pellets and sucrose pellets. It is pos- 
sible that this difference was partly responsible for the results, because two different pellets presumably have more in common than do a pellet and a solution. Consequently, their reinforcers would seem more likely to be encoded as separate events. It also should be noted, however, that the two different pellets used in the present study were always presented to two different locations (Colwill and Rescorla presented both reinforcers at the same location), and there was strong evidence from the pattern of responding at the end of compound-stimulus training that the two reinforcers were discriminated as separate events. Nevertheless, it is clear that additional evidence would be needed to establish that the reinforcers used here were clearly discriminated.

\section{EXPERIMENT 4}

A variety of previous experiments have shown that the identity of the reinforcer also plays a role in the effects of different contingencies of reinforcement. Typically, animals are trained on a schedule of response-dependent reinforcement, after which free reinforcers are superimposed on the operant baseline, with the result that the degree of suppression caused by the free reinforcers is substantially greater when the free reinforcer and responsecontingent reinforcer are the same versus when they are different (Colwill \& Rescorla, 1986; Dickinson \& Mulatero, 1989; Williams, 1989). To the extent that this effect can be demonstrated with the pair of reinforcers used in Experiments 1-3, clear evidence will be provided that the reinforcers are encoded as separate events. Experiment 4 was designed to provide this evidence.

\section{Method}

\section{Subjects}

The subjects were 16 male Sprague-Dawley albino rats obtained from Bantin Kingman. They ranged in age from 3 to 8 months at the start of the study, their experimental histories were similar to those of the subjects used in Experiment 1, and they were maintained as described in Experiment 1.

\section{Apparatus}

The apparatus was the same as that used in Experiment 1.

\section{Procedure}

Because all rats had acquired the leverpress response in a different apparatus, they were begun on the procedure without pretraining. A multiple schedule was presented in which 3-min components were regularly alternated, separated by 5 -sec time-out periods during which the contingencies were suspended. During the noise component, the white noise was continuously present, and presses of the left lever produced the reinforcer, when available. Presses of the right lever produced no reinforcers during the noise, but initiated a 5-sec change-over-delay (COD) contingency, which prevented responses to the left lever from being reinforced until at least $5 \mathrm{sec}$ had elapsed since the last incorrect response to the right lever. During the light component, the houselight located on the outside right side of the chamber was illuminated; presses of the right lever produced reinforcement and presses of the left lever initiated the 5-sec COD contingency for incorrect responding. The COD contingency was imposed after pilot work had in-
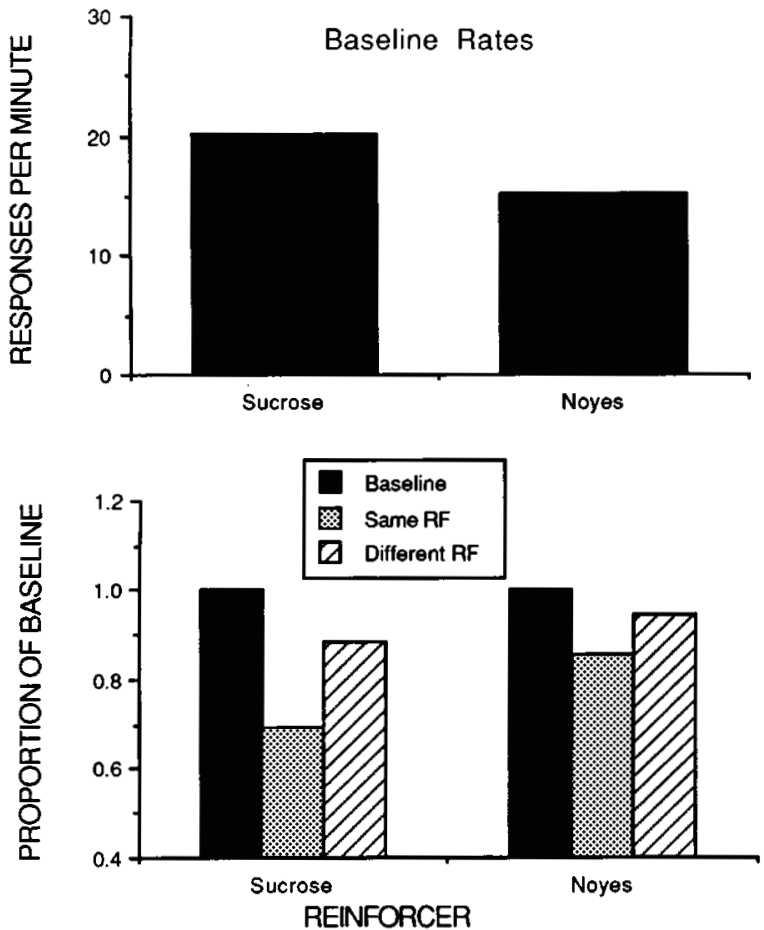

Figure 8. Results of Experiment 4. In the top panel are the response rates averaged over the last four sessions of both baseline conditions, separated according to which reward served as the reinforcer. In the bottom panel are the normalized response rates from the baseline and the two conditions in which free reinforcement was presented, again separated according to which reward served as the contingent reinforcer.

dicated that high rates of responding to the incorrect lever would otherwise occur.

During the first four sessions of training, the schedule governing reinforcement availability, which was always the same for the two components, was gradually increased from VI $15 \mathrm{sec}$ to VI $2 \mathrm{~min}$, where it remained for the duration of training. Sessions continued for a total of eight exposures to each component, or a total of $48 \mathrm{~min}$.

The procedure consisted of four phases of training. During Phases 1 and 4 (baseline and return to baseline), the response-contingent schedules were the only sources of reinforcement. Training during each of these phases continued for 16 sessions. During Phases 2 and 3, a VT 30-sec schedule of free reinforcement (VT $30 \mathrm{sec}$ ) was added. During one of these phases, the VT schedule delivered standard Noyes chow pellets; during the other phase, the VT schedule delivered sucrose pellets. The order of which reward served as the free reinforcer was counterbalanced over subjects.

The critical issue was whether the suppressive effects of the free reinforcer varied as a function of whether it was the same as or different from the response-contingent reinforcers. Thus, during Phases 2 and 3, the VT reinforcer was the same as the reinforcer for one of the responses but different from the other. For half of the subjects, sucrose pellets served as the reward for the left response and chow pellets as the rewards for the right response; for the remaining subjects, the contingencies were reversed. Training during Phases 2 and 3 continued for 20 sessions.

\section{Results}

The top portion of Figure 8 shows the mean response rates averaged over the last four sessions of both the ini- 
tial and final baseline conditions in which the free reinforcement schedule was not operating. The rates are presented in terms of which reward served as the reinforcer for a particular response, removing any role for the different response locations because of the counterbalancing of the lever-reward relationship across subjects. The mean response rate with sucrose as the reward was 20.1 responses/min, while the corresponding rate with Noyes chow pellets as the reinforcer was 15.1 responses $/ \mathrm{min}$. However, there was considerable variability across subjects in the pattern of reward preference, and this difference was not significant $[t(15)=1.68]$.

Because of the difference between the baseline response rates maintained by the two rewards, the effect of adding the free reinforcers was assessed by first normalizing the response rate during the conditions with the added free reinforcement relative to the baseline response rates for each subject. The mean response rate to each lever during the final four sessions of both baseline conditions was calculated for each subject, and those numbers were then divided into the response rates during the last eight sessions of each condition with the added VT schedules of free food. Thus, four normalized rates were obtained for each subject: for each of the two types of free reinforcer, the mean suppression was obtained for when the response-contingent reinforcer was the same as the free reinforcer and for when the response-contingent reinforcer was different from the free reinforcer.

The bottom portion of Figure 8 shows the mean suppression ratios subdivided according to which reinforcer served as the response-contingent reinforcer. The bars on the left show the response rates for the lever reinforced by sucrose pellets, first for the baseline condition without the free reinforcers, then with sucrose as the free reinforcer (same RF), and then with Noyes chow pellets as the free reinforcer (different RF). The bars on the right show the corresponding results for the lever reinforced by Noyes chow pellets. The data from the two free-reinforcer conditions were analyzed with a twofactor within-subject ANOVA, in which the identity of the contingent reinforcer was one factor, while the free reinforcer - same as or different from the contingent reinforcer - was the second factor. The identity of the contingent reinforcer was not significant $[F(1,15)=2.10]$. The effect of whether the reinforcer was the same as or different from the contingent reinforcer was significant $[F(1,15)=9.54]$. Thus, greater suppression occurred when the contingent reinforcer was the same as the free reinforcer than when the identities of the contingent and free reinforcers differed. It is noteworthy, from Figure 8, that this effect occurred even when the contingent reinforcer was Noyes chow pellets. Despite the sucrose being a more valued reinforcer, as indicated by the baseline response rates, suppression by the sucrose of the behavior maintained by the chow pellets was not as great as suppression by the free chow pellets themselves. The smaller difference between the same and the different reinforcement conditions seen on the right side of Figure 8 is presumably due to a combination of the overall value of the free reinforcer and its identity relative to that of the contingent reinforcer.

Averaged over the two types of contingent reinforcers, the normalized response rate when the free reinforcer was the same as the contingent reinforcer was .77, while the normalized response rate when the free reinforcer was different was .92. Separate $t$ tests on whether these values differed significantly from 1.0 showed that the value for the same-reinforcer condition was different from $1.0[t(15)=5.11]$, while the value for the different-reinforcer condition was not $[t(15)=1.23]$.

\section{Discussion}

Experiment 4 replicated the several previous experiments showing that less response suppression occurred when free reinforcers were added to a VI response-contingent baseline when the free and earned reinforcers had different identities than when they had the same identity. While this effect is clearly of interest in its own right, for the present purposes its importance is that it shows definitively that the chow and sucrose pellets were regarded as discriminatively different reinforcing events. Consequently, the failure of unblocking to occur in Experiments 1-3 cannot be ascribed to the use of reinforcers of insufficient discriminability.

\section{GENERAL DISCUSSION}

The present results are consistent with Ganesan and Pearce (1988) in showing that using a reinforcer in the compound-stimulus training phase that is different from that of the single-stimulus pretraining phase of a standard blocking design does not attenuate the degree of blocking that occurs. The results thus establish that their previous findings are not restricted to Pavlovian conditioning, but occur in instrumental conditioning as well. In fact, the procedure used by Ganesan and Pearce (1988) could itself be classified as instrumental in nature in that their conditioned response was approach to the food magazine conditional on the presence of an external cue, and it is not clear why such approach behavior should be functionally different from a discrete motor response such as barpressing (but see Balleine \& Dickinson, 1991, for an argument that magazine approach is a simple Pavlovian response).

The major puzzle posed by the present results lies in how they are to be reconciled with those of Rescorla (1990) and Colwill and Rescorla (1990), who found that changing the reinforcer contingencies correlated with a discriminative stimulus did prevent blocking from occurring. There is no obvious explanation for the conflict in the results, especially with respect to those of Experiment 3, whose procedures were designed to be very similar to those of Colwill and Rescorla. The only significant features of the present procedure that were not replications of their earlier work were the specific nature of the reinforcers used (they used a sucrose solution vs. chow pellets in contrast to the present sucrose pellets vs. 
chow pellets), and the location of the reinforcer deliveries (they presented both reinforcers at the same site; here two different sites were employed). It is perhaps worthwhile to consider the possible effects of each of these differences.

The specific nature of the different reinforcers employed potentially determines the likelihood of their being encoded as separate events. It is possible, therefore, that the present pair of reinforcers was not sufficiently different for such differential encoding to occur. This interpretation is challenged, however, by the results of Experiment 4, in which strong effects of the reinforcer identity were obtained in the contingency procedure in which free reinforcers were superimposed on a responsecontingent baseline. When the free reinforcers were different from the contingent reinforcer, substantially less response suppression occurred. The issue thus becomes the difference between the reinforcers: why was it sufficient to produce differential effects of reinforcer identity in the contingency procedure but not in the blocking procedure? Perhaps the contingency procedure is simply more sensitive, or perhaps different processes underlie the two types of effects.

The hypothesis that the present failure to produce unblocking was due to insufficient discriminability between the two reinforcers is also challenged by the earlier results of Ganesan and Pearce (1988). Their different reinforcers were food versus water, surely as discriminably different as any possible pair of appetitive reinforcers, and yet they too failed to produce the unblocking effect.

The use of two different reinforcer locations in the present study also was potentially a factor in the discrepancy between the present findings and previous work. At first glance, it would seem that different reinforcer sites for the two reinforcers should have enhanced, rather than decreased, the chances of producing unblocking, because differences in spatial location should add to the differences in reinforcer quality as a basis for the subject's being "surprised" by the change in reinforcers at the start of compound stimulus training. This possibility is supported by the results of Ganesan and Pearce (1988), who used different reinforcer locations in their last experiment, which produced a marginally significant unblocking effect, in contrast to the complete failure to produce unblocking in earlier experiments using a single reinforcer location. However, differences in reinforcer location might have had other effects that obscured the measurement of any unblocking effects that actually occurred. One possibility is greater response competition. Accordingly, subjects without a change in reinforcer between the pretraining and compound stimulus phase would interrupt their barpressing with visits to a single feeder site, while those experienced with two different reinforcer sites during the different elements of the compound stimulus might visit both sites before returning to barpressing. The plausibility of greater response competition from two different reinforcer locations is supported by the lower response rates that were maintained at the end of the compound stimulus training to the compound stimulus associated with the change in reinforcer. Perhaps this greater degree of response competition reduced response rate sufficiently to obscure the increase in response rate to that compound stimulus that should have occurred given the unblocking effect. This possibility seems implausible, however, because the size of the response-rate difference at the end of training was relatively small, while the blocking effect itself was very large. In addition, the same differences in response competition should also have occurred in the contingency manipulation of Experiment 4, causing the stimulus correlated with different reinforcer identities for the contingent versus free reinforcer to evoke response competition with barpressing that was greater than that evoked by the stimulus correlated with the same reinforcer. Thus, response rates should have been reduced more to the former than to the latter. The opposite effect occurred, with greater response suppression when the free and contingent reinforcers had the same identity. Thus, differences in response competition caused by two different reinforcer sites seems an unlikely explanation of the present results unless the role of response competition is assumed to be different for the blocking versus contingency procedures.

A second possible way that the use of two different reinforcer locations could have been important is that position was a more salient cue than reinforcer quality, and thus overshadowed reinforcer quality in the manner in which the reinforcer identity was encoded. Then, for whatever reason, a change in reinforcer location was not itself sufficiently "surprising" to produce the unblocking effect. Again, however, this explanation is weakened by the occurrence of reinforcer identity effects in the contingency procedure of Experiment 4, and by the results of Ganesan and Pearce (1988), which showed a tendency for unblocking that was greater with two different reinforcer sites than with the same site for both reinforcers.

One possible resolution to the conflicting results may lie, at least partly, in the different measures used to assess the degree of blocking. In the procedures of Colwill and Rescorla, within-subject designs were used in which the degree of stimulus control was compared for stimuli that were correlated with the same versus different reinforcer during the compound-stimulus phase relative to that paired with the pretrained stimulus. No control conditions were included for the degree of stimulus control by a stimulus paired with the reinforcer without a history of pretraining for the compounded stimulus. Thus, it is possible that a large amount of blocking occurred to both stimuli, with some additional effect of changes in the reinforcer that reduced somewhat the degree of blocking for the stimulus paired with the changed reinforcer. In other words, changes in the reinforcer identity may be a relatively weak manipulation in comparison to the magnitude of blocking effects that typically occur. Depending upon the exact nature of the different reinforcers (the degree of their shared properties), it may or may not be ev- 
ident, depending on the sensitivity of the measurement procedure.

It is clear from the above discussion that the determinants of when blocking or unblocking will occur remain largely unspecified. The simple notion of "surprise" seems an unlikely candidate for explaining the discrepant findings, since clear evidence of discriminability of the change in reinforcer occurred even when no unblocking occurred. Also unclear is how the failure to obtain unblocking is to be related to the specificity of the response-reinforcer association that has been shown in the reinforcer devaluation experiments (e.g., Colwill \& Rescorla, 1986). The array of previous findings suggests that reinforcer identity plays a very different role in the two types of experiments, a disparity that suggests caution in using either set of data for a general interpretation of the nature of what is learned in instrumental conditioning.

The difficulty of understanding the role of reinforcer identity is not restricted to the study of instrumental conditioning. Previous findings on the effects of changes in reinforcer value on Pavlovian second-order conditioning have revealed a similar complexity. In these experiments one stimulus (S1) was paired with the reinforcer; then a second stimulus (S2) was paired with S1. When S1 was separately extinguished, the issue was whether the behavior to S2 would be affected by S1 extinction without additional S2-S1 pairings. Early research using both aversive (Rizley \& Rescorla, 1972) and appetitive (Holland \& Rescorla, 1975b) procedures showed no effect of the change in value of S1, while subsequent research using the autoshaping procedure showed a strong reduction in S2 responding after separate extinction of S1 (Rashotte, Griffin, \& Sisk, 1977). A resolution to this conflict was offered by Nairne and Rescorla (1981), who noted that the first pattern of results could be obtained in the autoshaping procedure in which $\mathrm{S} 1$ was a noise while the latter pattern of results occurred when S1 was a keylight (S2 was always a keylight). Nairne and Rescorla thus proposed that the similarity between $\mathrm{S} 1$ and $\mathrm{S} 2$ determined which attributes of S1 were associated with S2, which in turn determined whether the change in S1 value affected the value of S2.

The assumption that reinforcing events have multiple attributes that vary in their likelihood of association with other associative elements can of course be extended to instrumental conditioning. Accordingly, the role of changes in the reinforcer, either in terms of its identity in blocking procedures or in terms of its value in devaluation procedures, cannot be predicted easily because there is no way of determining in advance which reinforcer attributes will be involved. Selective encoding of different attributes of the reinforcer is more evident with second-order than with first-order conditioning, given that reinforcer devaluation after first-order conditioning has generally affected the level of conditioned responding (e.g., Holland \& Rescorla, 1975a) despite the fact that the CS and US (or response and reinforcer in instrumental conditioning) are distinctly different (e.g., a light vs. shock or a leverpress vs. a food pellet). This possibility only adds to the complexity of the analysis of any particular instrumental conditioning procedure, however, because it is typically ambiguous whether the operant response is under the direct control of the primary reinforcer (e.g., food) or of the stimulus change that precedes the delivery of food (e.g., the click of the pellet dispenser). To the extent that conditioned reinforcement effects play a significant role in maintaining operant behavior, the prior literature on second-order conditioning suggests that it should not be surprising that changes in the reinforcer should have little impact.

A final aspect of the results worth noting is the sensitivity of the contingency manipulation in Experiment 4 to the identity of the reinforcer, in contrast to the blocking studies in which changing the identity of the reinforcer had little effect. The dissociation of the two procedures with respect to the reinforcer-identity variable, even when the same pair of reinforcers and same responses were used in both cases, challenges explanations of contingency effects in terms of "context blocking" (see Durlach, 1989, for a review). That is, the decrement caused by the addition of the free reinforcers has been assumed to be because the background cues become associated with the reinforcer and thus provide associative competition with the response for association with the reinforcer. According to such an explanation, the effects of reinforcer identity in contingency experiments should be similar to those in blocking experiments, which was not the case here. Given the conflict in results obtained with different experiments on blocking itself, it is premature to draw strong conclusions about the importance of this dissociation. The finding that blocking and contingency effects do not necessarily obey similar functional relations is nevertheless provocative.

\section{REFERENCES}

BaKal, C. W., Johnson, R. D., \& Rescorla, R. A. (1974). The effect of change in US quality on the blocking effect. Pavlovian Journal of Biological Science, 9, 97-103.

BALleine, B. W., \& Dickinson, A. (1991). Instrumental performance following reinforcer devaluation depends upon incentive learning. Quarterly Journal of Experimental Psychology, 43B, 279-296.

Colwill, R. M., \& Rescorla, R. A. (1986). Associative structures in instrumental learning. In G. H. Bower (Ed.), The psychology of learning and motivation (Vol. 20, pp. 55-104). New York: Academic Press.

Colwill, R. M., \& Rescorla, R. A. (1990). Evidence for the hierarchical structure of instrumental learning. Animal Learning \& Behavior, 18, 71-82.

Delamater, A. R., \& LoLordo, V. M. (1991). Event revaluation procedures and associative structures in Pavlovian conditioning. In L. Dachowski \& C. F. Flaherty (Eds.), Current topics in animal learning: Brain, emotion, and cognition (pp. 55-94). Hillsdale, NJ: Erlbaum.

Dickinson, A., Hall, G., \& Mackintosh, N. J. (1976). Surprise and the attenuation of blocking. Journal of Experimental Psychology: Animal Behavior Processes, 2, 313-322.

Dickinson, A., \& Mulatero, C. W. (1989). Reinforcer specificity of the suppression of instrumental performance on a non-contingent schedule. Behavioural Processes, 19, 2.

Durlach, P. (1989). Learning and performance in Pavlovian conditioning: Are failures of contiguity failures of learning or perfor- 
mance? In S. B. Klein \& R. R. Mowrer (Eds.), Contemporary learning theories: Pavlovian conditioning and the status of traditional learning theory (pp. 19-59). Hillsdale, NJ: Erlbaum.

Ganesan, R., \& Pearce, J. M. (1988). Effect of changing the unconditioned stimulus on appetitive blocking. Journal of Experimental Psychology: Animal Behavior Processes, 14, 280-291.

Holland, P. C. (1988). Excitation and inhibition in unblocking. Journal of Experimental Psychology: Animal Behavior Processes, 14, 261-279.

Holland, P. C., \& Rescorla, R. A. (1975a). The effect of two ways of devaluing the unconditioned stimulus after first- and secondorder appetitive conditioning. Journal of Experimental Psychology: Animal Behavior Processes, 1, 355-363.

Holland, P. C., \& Rescorla, R. A. (1975b). Second-order conditioning with food unconditioned stimulus. Journal of Comparative \& Physiological Psychology, 88, 459-467.

KAMIN, L. J. (1968). Attention-like processes in classical conditioning. In M. R. Jones (Ed.), Miami Symposium on the Prediction of Behavior: Aversive stimulation (pp. 9-32). Coral Gables, FL: University of Miami Press.

KonorsKI, J. (1967). Integrative activity of the brain. Chicago: University of Chicago Press.

Mackintosh, N. J., \& CotTon, M. M. (1985). Conditioned inhibition for reinforcement reduction. In R. R. Miller \& N. E. Spear (Eds.), Information processing in animals: Conditioned inhibition (pp. 89111). Hillsdale, NJ: Erlbaum.

NaIRne, J. S., \& Rescorla, R. A. (1981). Second-order conditioning with diffuse auditory reinforcers in the pigeon. Learning \& Motivation, 12, 65-91.

Rashotte, M. E., Griffin, R. W., \& Sisk, C. L. (1977). Second-order conditioning of the pigeon's keypeck. Animal Learning \& Behavior, 5, 25-38.

ResCorla, R. A. (1990). The role of information about the responseoutcome relation in instrumental discrimination learning. Journal of Experimental Psychology: Animal Behavior Processes, 16, 262270.

Rizley, R. C., \& Rescorla, R. A. (1972). Associations in secondorder conditioning and sensory preconditioning. Journal of Comparative \& Physiological Psychology, 81, 1-11.

StiCKNeY, K. J., \& Donahoe, J. W. (1983). Attenuation of blocking by a change in US locus. Animal Learning \& Behavior, 11, 60-66.

WAGNER, A. R., \& BRANDON, S. E. (1989). Evolution of a structured connectionist model of Pavlovian conditioning (AESOP). In S. B. Klein \& R. R. Mowrer (Eds.), Contemporary learning theories: Pavlovian conditioning and the status of traditional learning theory (pp. 149-189). Hillsdale, NJ: Erlbaum.

Wagner, A. R., Mazur, J. E., Donegan, N. H., \& Pfautz, P. L. (1980). Evaluation of blocking and conditioned inhibition to a CS signaling a decrease in US intensity. Journal of Experimental Psychology: Animal Behavior Processes, 6, 376-385.

WiLliams, B. A. (1989). The effects of response contingency and reinforcement identity on response suppression by alternative reinforcement. Learning \& Motivation, 20, 204-224.

Williams, B. A., Preston, R. A., \& De Kervor, D. (1990). Blocking of the response-reinforcer association: Additional evidence. Learning \& Motivation, 21, 379-398.

(Manuscript received August 30, 1993; revision accepted for publication April 2, 1994.) 